\title{
Connection between HPS and ACLF: a solution of chaos?
}

\author{
Hitoshi Maruyama ${ }^{1}\left[\right.$. Shuichiro Shiina ${ }^{1}$
}

Received: 24 July 2021 / Accepted: 11 September 2021 / Published online: 4 October 2021

(c) Asian Pacific Association for the Study of the Liver 2021

Hepatopulmonary syndrome (HPS) is classified as a pulmonary vascular disorder that occurs in liver disease and/or portal hypertension and is characterized by intrapulmonary vascular dilatation with arterial deoxygenation. Nitric oxide (NO) is a key factor for systemic and pulmonary vasodilatation and plays a certain role in the hyperdynamic circulatory state in the patients. It is the consequence of abnormal angiogenesis of the pulmonary microcirculation [1]; distal vessels are involved in remodeling, including dilated precapillary and capillary vessels and precapillary arteriovenous communications [2]. HPS is detected in acute/ chronic liver diseases irrespective of the presence/absence of portal hypertension; the prevalence is approximately $10 \%$ in chronic viral hepatitis with or without cirrhosis, $18 \%$ in orthotopic liver transplantation candidates and as high as $28 \%$ in Budd-Chiari syndrome [3, 4]. Cirrhosis is the common liver disease leading to HPS.

A prospective study in 111 cirrhotic patients reported that the mortality of patients with HPS was significantly higher (median survival, 10.6 months) than the mortality of patients without HPS (40.8 months, $p<0.05$ ), even after adjusting for the severity of liver disease ( 2.9 months in patients with HPS $[n=15]$ and 14.7 months without HPS $[n=35, p<0.05]$ in Child-Pugh class C) [5]. The presence of HPS was also an independent predictor of survival by multivariate analysis, and mortality correlated with the severity of HPS. Therefore, the presence of HPS seems to be an independent and significant factor for the prognosis of cirrhotic patients.

The development of portosystemic collaterals, reduced liver function typified by the reduction of the intrahepatic phagocytosis and the occurrence of bacterial translocation in cirrhosis, provide inflammatory and angiogenic cytokines, circulating bacteria or bacterial endotoxins to enter the pulmonary circulation, damage the pulmonary endothelium and

Hitoshi Maruyama

h.maruyama.tw@juntendo.ac.jp

1 Department of Gastroenterology, Juntendo University, 2-1-1, Hongo, Bunkyo-ku, Tokyo 113-8421, Japan induce extensive recruitment of pulmonary intravascular macrophages $[6,7]$. Recent basic studies suggest that bacterial translocation and/or proinflammatory cytokines such as tumor necrosis factor-a, interleukin-1 (IL-1) and IL-8, which are upregulated in the lung and pulmonary intravascular macrophages, are involved in the pathogenesis of HPS [8-10].

Acute-on-chronic liver failure (ACLF) is considered a distinct clinical syndrome that is demonstrated among patients with acutely decompensated chronic liver disease and/or cirrhosis. ACLF is characterized by an intense systemic inflammatory response, single- or multiple organ system failures, and a high 28-day mortality rate [11], although detailed definitions differ according to the different international criteria, Europe, North America, Asian Pacific region, and China [12]. The poor prognosis of ACLF is generally recognized; transplantation-free mortality rates were $32.8 \%$ at 28 days and $51.2 \%$ at 90 days in patients with ACLF and $1.8 \%$ at 28 days and $9.8 \%$ at 90 days in those without ACLF [13]. With the increased prevalence of nonalcoholic fatty liver disease (NAFLD) worldwide, a recent study from the United States based on the survey between 2005 and 2017 has shown the waitlist registrants for NAFLD-ACLF rose by $331.6 \%$ from 134 to 574 candidates $(p<0.001)$, representing the largest percentage increase in the study population [14]. They suggested that patients with NAFLD-ACLF will likely have the highest risk of waitlist mortality.

Against this background, $\mathrm{Ki}$ et al. conducted a longitudinal prospective observational study in 142 patients with cirrhosis (median follow-up period, 28 months), all of whom underwent saline-agitated contrast echocardiography for the diagnosis of HPS in South Korea from 2014 to December 2019 [15]. In the basis of diagnosis, unfortunately, the definition/criteria of ACLF and HPS are not clearly described in the manuscript. As for the former, the authors might have used the Asian Pacific Association for the Study of the Liver (APASL) definition as a member of Asian countries, acute hepatic insult manifesting as jaundice (total bilirubin levels of $5 \mathrm{mg} / \mathrm{dl}$ or more) and coagulopathy (international normalized ratio $>-1.5$, or prothrombin activity $<40 \%$ ) 
complicated within 4 weeks by clinical ascites, hepatic encephalopathy, or both [16]. Also, as for the latter, international liver transplant society diagnostic and management guidelines based on European respiratory society task force on pulmonary-hepatic disorders is recognized as a reference standard [17].

First, Ki et al. found that cirrhosis patients diagnosed with HPS had a poor prognosis, which is not new and in agreement with previous data [5]. However, the next data appeared to be novel and attractive; the HPS group had a significantly higher incidence of ACLF than the nonHPS group (13 cases [15.6\%] in the nonHPS group and 24 cases [40.6\%] in the HPS group, $p<0.01)$ and more frequently presented with lung involvement of ACLF ( $p=0.03$ ), suggesting that HPS is a significant risk factor for ACLF. The results may have the potential to alter the practical management of cirrhosis patients with HPS to be much more careful.

In general, there may be two reasons to explain HPSrelated organ failure. One is the presence of hypoxemia, which may cause multiorgan ischemic damage. The second is extrapulmonary abnormalities due to right-to-left intrapulmonary communications, supported by reports regarding the development of a brain abscess or intracranial hemorrhage and hypoxemia-induced polycythemia [3]. However, according to the study by Ki et al., the severity of HPS had effects neither on mortality in the HPS group nor on ACLF development, although Child-Turcotte-Pugh (CTP, class B/C) and model for end-stage liver disease (MELD) score $\geq 18$ were identified as risk factors for mortality in the HPS group (CTP class $\mathrm{B} / \mathrm{C}$, hazard ratio $[\mathrm{HR}]=1.75,95 \%$ confidence interval [CI] 0.6-5.0, $p=0.29$; MELD score $\geq 18 \mathrm{HR}=2.51$, $95 \%$ CI $1.32-4.8, p<0.01)$ [15]. The data seem against the influence of the two factors, the presence of hypoxemia and right-to-left intrapulmonary communications, as the cause of ACLF. Meanwhile, there are some concerns which need to be considered when we interpret the data presented by $\mathrm{Ki}$ et al. First, as the severity of HPS is typically defined by $\mathrm{PaO}_{2}$ levels and the echocardiographic criteria seem subjective, more detailed explanation why the severity of HPS had no impact on mortality may be necessary. Second, CTP B/C cannot be labeled as a risk factor for mortality in HPS group if the $95 \% \mathrm{CI}$ is $0.6-5.0$, with $p=0.29$. Third, the number of patients with CTP A was 30 in nonHPS group and 11 in HPS group, and that of patients with CTP B/C was 53 in nonHPS group and 48 in HPS group, showing significant difference $(p=0.0233)$. This may be responsible for more chances of ACLF in HPS group rather than HPS itself. Point is, due to the potential bias in the cohort, significance of HPS as a risk factor for ACLF needs to be proven by the careful analysis to rule out the impact of confounding factors.

What factors affect developing ACLF in the possible pathophysiological mechanisms of HPS? The most likely cause may be infection, as $\mathrm{Ki}$ et al. also mentioned in the manuscript [15]. Systemic inflammation plays a major role in the pathophysiology of both HPS and ACLF [1, 2, 11]; patients with ACLF have intense systemic inflammation and oxidative stress, unlike patients who have acute decompensation but no organ failure [18-20]. Furthermore, the evidence has shown that systemic inflammation correlates well with the severity of ACLF: the greater the intensity of systemic inflammation is the larger the number of organ failures at enrollment and the higher the short-term mortality [11]. However, this is just one of the possible reasons, and the basic mechanism may not be simple.

The change in hepatic condition from compensated status to decompensation/ACLF may be explained by the combination of predisposing, precipitating, and perpetuating factors (Fig. 1). The following conditions are known as predisposing factors: dysbiosis, immune dysfunction, alternation of serum albumin, and genetic and epigenetic predisposition [12]. Dysbiosis is characterized by qualitative and quantitative alteration of the microbiome and is linked to intestinal barrier dysfunction and increased intestinal permeability, leading to bacterial translocation (called pathogen-associated molecular patterns). Dysbiosis is also affected by proton pump inhibitors.

Next, cirrhosis is involved in immunodeficiency and persistent proinflammatory immune cell activation, which result in immune exhaustion, leading to immune dysfunction. Cirrhosis accounts for hemodynamic changes and worsening of portal hypertension via systemic inflammation and stimulates the transition to decompensation and organ failure. Alternation of transcriptional and epigenetic profiles presented by downregulation of key innate immune and metabolic pathways accompanied by the upregulation of immune-related systems responsible for immune exhaustion may be closely linked to CD14+ monocytes in patients with alcoholic hepatitis [21].

Oxidative damage is known to impair the binding properties of albumin, and the reduced binding capacity of albumin site II is related mainly to impaired liver function in advanced liver disease [22]. A study performed later reported that oxidized albumin triggers a cytokine storm in leukocytes through P38 mitogen [23]. Taken together, the oxidation of albumin is closely associated with disease progression in cirrhosis and may be predictive of their prognosis. Elevation of ischemia-modified albumin may also be linked with disease severity and may play a prognostic role in ACLF [24]. Regarding genetics, although there may be a potential impact on gene polymorphisms, this field is just in the research stage, and there are only a few studies; sodium dismutase polymorphisms [25] and the PNPLA3 G/G genotype may be associated with an increased risk of decompensation [26].

Many factors are known as precipitating factors that enhance the transition to acute decompensation/ACLF: 


\section{Predisposing factors}

\begin{tabular}{c|} 
Dysbiosis \\
Immune dysfunction \\
Genetic predisposition \\
Epigenetic predisposition \\
Alternation of serum albumin
\end{tabular}

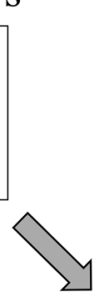

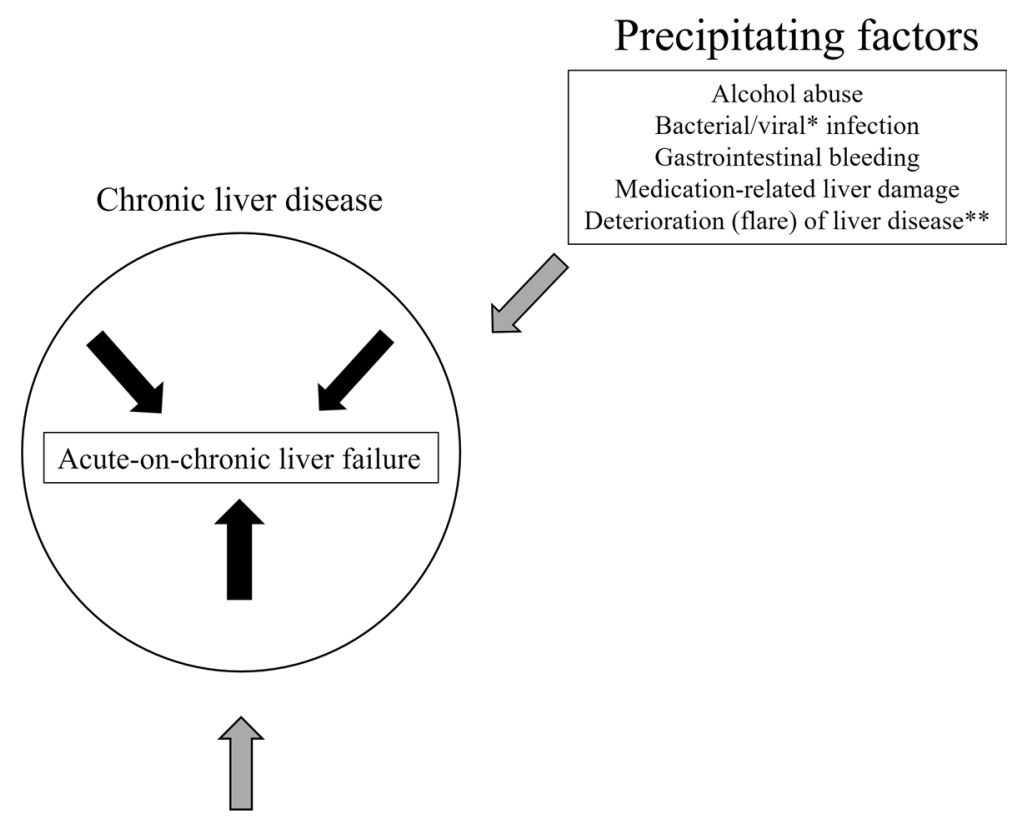

Perpetuating factors

Combination of predisposing/precipitating factors

Fig. 1 Existing mechanisms of developing acute-on-chronic liver failure. *Hepatotropic viruses (hepatitis $\mathrm{A}$ and $\mathrm{E}$ virus) and other viruses (cytomegalovirus, Epstein Barr virus, SARS-CoV-2, and COVID-19).

bacterial infection, alcohol, gastrointestinal bleeding typified by variceal bleeding, medication-related liver damage, deterioration (flare) of the disease such as autoimmune hepatitis, hepatitis B-related liver disease, and Wilson's disease, and acute infection with hepatotropic viruses (hepatitis A and $\mathrm{E}$ virus) and other viruses (cytomegalovirus, Epstein Barr virus, SARS-CoV-2, and COVID-19). Perpetuating factors may be single or multiple events from predisposing/ precipitating factors, showing a vicious circle. Thus, further studies may be needed to clarify the mechanism to develop ACLF through HPS.

Based on these complex backgrounds, many attempts have been made for the microbiome as a therapeutic target: improvement of dysbiosis and suppression of bacterial translocation using fecal microbial transplantation, antibiotics, probiotics, prebiotics, and short-chain fatty acids [27]. Based on the principal pathophysiology of HPS, bacterial overgrowth may be the axis of therapeutic target to prevent bacterial translocation which may have a potential to control HPS by suppressing NO synthesis. However, against the animal study showing positive results in decreasing bacterial translocation, pulmonary macrophages, and the activity of NO synthase, a pilot randomized crossover clinical trial of norfloxacin did show no effect to improve HPS [28]. Validation of substantial role
**Liver diseases such as autoimmune hepatitis, hepatitis B virusrelated liver disease, and Wilson's disease

of the medication remains to be elucidated by larger multicenter randomized trials. As for the future perspective, it may be necessary to detect specific marker for both HPS and ACLF, and the predictive factor for developing them. In addition, it should be examined whether the treatment for HPS may reduce the occurrence of ACLF and improve the clinical outcome.

In conclusion, the study by $\mathrm{Ki}$ et al. demonstrated the relationship between HPS and ACLF in such a complicated mechanism to ACLF [14]. However, the connection is also far from the solution, although the process may have taken a step forward in elucidating the pathophysiology to the transition to ACLF. There is still a chaotic problem in the development of ACLF; therefore, continuous efforts should be needed in the future.

Funding None.

\section{Declarations}

Conflict of interest Hitoshi Maruyama and Shuichiro Shiina have declared that there are no conflicts of interest.

Ethical approval This article does not contain any studies with human participants or animals performed by any of authors. 


\section{References}

1. Herve $\mathrm{P}$, Lebrec D, Brenot F, et al. Pulmonary vascular disorders in portal hypertension. Eur Respir J 1998;11:1153-1166

2. Rodriguez-Roisin R, Agusti AG, Roca J. The hepatopulmonary syndrome: new name, old complexities. Thorax 1992;47:897-902

3. Rodriguez-Roisin R, Krowka MJ, Herve P, et al. Pulmonaryhepatic vascular disorders (PHD). Eur Respir J 2004;24:861-880

4. Martinez GP, Barbera JA, Visa J, et al. Hepatopulmonary syndrome in candidates for liver transplantation. J Hepatol 2001;34:651-657

5. Schenk P, Schoniger-Hekele M, Fuhrmann V, et al. Prognostic significance of the hepatopulmonary syndrome in patients with cirrhosis. Gastroenterology 2003;125:1042-1052

6. Maruyama H, Shiina S. Collaterals in portal hypertension: anatomy and clinical relevance. Quant Imaging Med Surg 2021;11:3867-3881

7. Nunes H, Lebrec D, Mazmanian M, et al. Role of nitric oxide in hepatopulmonary syndrome in cirrhotic rats. Am J Respir Crit Care Med 2001;164:879-885

8. Rabiller A, Nunes H, Lebrec D, et al. Prevention of gram-negative translocation reduces the severity of hepatopulmonary syndrome. Am J Respir Crit Care Med 2002;166:514-517

9. Sztrymf B, Rabiller A, Nunes H, et al. Prevention of hepatopulmonary syndrome and hyperdynamic state by pentoxifylline in cirrhotic rats. Eur Respir J 2004;23:752758

10. Sztrymf B, Libert JM, Mougeot C, et al. Cirrhotic rats with bacterial translocation have higher incidence and severity of hepatopulmonary syndrome. J Gastroenterol Hepatol 2005;20:1538-1544

11. Arroyo V, Moreau R, Jalan R. Acute-on-chronic liver failure. N Engl J Med 2020;382:2137-2145

12. Moreau R, Gao B, Papp M, et al. Acute-on-chronic liver failure: a distinct clinical syndrome. J Hepatol 2021;75(Suppl 1):S27-S35

13. Moreau R, Jalan R, Ginès $P$, et al. Acute-on-chronic liver failure is a distinct syndrome that develops in patients with acute decompensation of cirrhosis. Gastroenterology 2013;144:1426-1437

14. Sundaram V, Jalan R, Shah $P$, et al. Acute on chronic liver failure from nonalcoholic fatty liver disease: a growing and aging cohort with rising mortality. Hepatology 2021;73:1932-1944

15. Ki HS, Kim MY, Kang SH, et al. Hepatopulmonary syndrome is related to the development of acute-on-chronic liver failure and poor prognosis in cirrhotic patients. Hepatol Int (in press)

16. Sarin SK, Choudhury A, Sharma MK. Acute-on-chronic liver failure: consensus recommendations of the Asian Pacific association for the study of the liver (APASL): an update. Hepatol Int 2019;13:353-390

17. Krowka MJ, Fallon MB, Kawut SM, et al. International liver transplant society practice guidelines: diagnosis and management of hepatopulmonary syndrome and portopulmonary hypertension. Transplantation 2016;100:1440-1452

18. Clària J, Stauber RE, Coenraad MJ, et al. Systemic inflammation in decompensated cirrhosis: characterization and role in acute-onchronic liver failure. Hepatology 2016;64:1249-1264

19. Bernsmeier C, Pop OT, Singanayagam A, et al. Patients with acute-on-chronic liver failure have increased numbers of regulatory immune cells expressing the receptor tyrosine kinase MERTK. Gastroenterology 2015;148:603-615

20. Fernández J, Acevedo J, Wiest R, et al. Bacterial and fungal infections in acute on-chronic liver failure: prevalence, characteristics and impact on prognosis. Gut 2018;67:1870-1880

21. Weichselbaum L, Azouz A, Smolen KK, et al. Epigenetic basis for monocyte dysfunction in patients with severe alcoholic hepatitis. J Hepatol 2020;73:303-314

22. Oettl K, Birner-Gruenberger R, Spindelboeck W, et al. Oxidative albumin damage in chronic liver failure: relation to albumin binding capacity, liver dysfunction and survival. J Hepatol 2013;59:978-983

23. Alcaraz-Quiles J, Casulleras M, Oettl K, et al. Oxidized albumin triggers a cytokine storm in leukocytes through P38 mitogen-activated protein kinase: role in systemic inflammation in decompensated cirrhosis. Hepatology 2018;68:1937-1952

24. Jalan R, Schnurr K, Mookerjee RP, et al. Alterations in the functional capacity of albumin in patients with decompensated cirrhosis is associated with increased mortality. Hepatology 2009;50:555-564

25. Schwab S, Lehmann J, Lutz P, et al. Influence of genetic variations in the SOD1 gene on the development of ascites and spontaneous bacterial peritonitis in decompensated liver cirrhosis. Eur J Gastroenterol Hepatol 2017;29:800-804

26. Mandorfer M, Scheiner B, Stättermayer AF, et al. Impact of patatin-like phospholipase domain containing 3 rs738409 G/G genotype on hepatic decompensation and mortality in patients with portal hypertension. Aliment Pharmacol Ther 2018;48:451-459

27. Tranah TH, Edwards LA, Schnabl B, et al. Targeting the gut-liverimmune axis to treat cirrhosis. Gut 2021;70:982-994

28. Gupta S, Faughnan ME, Lilly L, et al. Norfloxacin therapy for hepatopulmonary syndrome: a pilot randomized controlled trial. Clin Gastroenterol Hepatol 2010;8:1095-1098

Publisher's Note Springer Nature remains neutral with regard to jurisdictional claims in published maps and institutional affiliations. 